\title{
LEPTON BEAM POLARIZATION AT LEP
}

R. Assmann*, A. Blondel*, B. Dehning, A. Drees, P. Grosse-Wiesmann, H. Grote, R. Jacobsen ${ }^{\circ}$, J.-P. Koutchouk, J. Miles, M. Placidi, R. Schmidt, F. Tecker ${ }^{\dagger}$, J. Wenninger

\author{
CERN, CH-1211 Geneva 23, Switzerland \\ * SLAC, Stanford Univ., P.O.Box 4349, CA 94309, USA \\ * Ecole Polytechnique, Paris, France \\ - Dept. of Physics, U.C. Berkeley, CA 94720, USA \\ $\dagger$ Physikalisches Institut III A, RWTH Aachen, Germany
}

\begin{abstract}
Results from studies on transverse polarization in LEP over the past two years are presented. A single beam transverse polarization level of $57 \%$ at $45 \mathrm{GeV}$ was reached adopting strategies to compensate depolarizing effects originating in the four experimental solenoids and from orbit perturbations. Beam Energy Calibration was performed by Resonant Depolarization during the 1993 LEP Run for Physics at three different energies centered around the $Z$ peak. The uncertainty on the beam energy was reduced to about $1 \mathrm{MeV}$, thus improving the accuracy on the Z-resonance mass and width with respect to previous results. Successful results obtained at the end of the 1994 LEP Run on polarization with colliding beams are reported and future plans outlined.
\end{abstract}

\section{INTRODUCTION}

The experiments conceived and performed in the last two years aimed at producing beam polarization in conditions similar to luminosity operation and at developing orbit correction methods capable of increasing the polarization level at the $Z$ energies and beyond [1].

The accuracy and reliability of the beam position monitors was greatly improved for this purpose. A vertical realignment of the magnetic structure of LEP was carried out and proved very beneficial.

Polarization during luminosity operation required compensating the spin precession in the Experimental Solenoids and studies on the effects of the machine tunes [2],[3].

The spin resonance compensation method known as Harmonic Spin Matching [4] was implemented and improved [5] providing high single beam polarization level.

Polarization with colliding beams was obtained in two dedicated machine development sessions with a proper choice of the beam energy and by controlling the number of collisions per revolution of the circulating bunches.

\section{SINGLE BEAM POLARIZATION STUDIES}

\subsection{Solenoid COMPEnsation}

Precise Energy Calibration during physics runs requires polarization in presence of the very strong longitudinal magnetic fields produced by the experimental solenoids. The spin rotation around the longitudinal axis (up to $66 \mathrm{mrad}$ for the $10 \mathrm{Tm}$ strength of the ALEPH solenoid) can be compensated by a proper configuration of vertical closed orbit bumps at both sides of each solenoid. The spin axis of the incoming beam is on purpose counterrotated out of the vertical direction by half of the rotation angle produced by the magnet by the closed bumps at the entrance of the solenoid. An identic bump configuration at the other side of the solenoid makes a closed-spin-bump antisymmetric w.r.t. the IP and compensates for the spin rotation in the second half of the magnet. 
The initial scheme foreseen for the 1991 LEP optics (60 degree lattice) [2] was adapted to the recent LEP optics developments [3] and provided substantial improvement in the attained polarization level.

\subsection{Constraints from the tunes}

Strong depolarizing processes occur when the spin precession is in resonance with synchrotron and betatron oscillations.

The synchrotron tune $Q_{s}$ was adjusted with the criterion of making the satellites from the integer spin tunes above and below the fractional one to coincide, which defined a polarization $Q_{s}=0.0625$.

The integer part of the betatron tunes produces systematic integer resonances [6]:

$$
\left(\operatorname{Int}\left[\nu_{\mathrm{s}}\right]\right)_{\text {syst }} \equiv N_{\text {syst }}=4 \cdot k \pm \operatorname{Int}\left[\mathrm{Q}_{\mathrm{x}}, \mathrm{Q}_{\mathrm{y}}\right]
$$

which, at the present vertical tune $\left(Q_{y}=76 . \mathrm{xx}\right)$ generate "unwanted lines" dangerous at PEAK CM energy $\left(\nu_{s} \sim 103.5\right)$ but in principle less important at PEAK \pm 2 CM energies (LEP scan campaign in 1993).

The fractional part of the betatron tunes intervenes in the $Q_{x}+Q_{y}$ resonance driven by off-axis orbits in the sextupoles. For the values of the betatron tunes used in physics the above resonance is very close to the fractional part of the spin tune at which most of the calibrations take place.

To avoid these unwanted effects the machine tunes for polarization studies were :

$$
\left(q_{x}\left|q_{y}\right| Q_{s}\right)_{P_{\perp}}=0.10|0.16 \div 0.20| 0.0625
$$

\subsection{Spin Diffusion}

In a perfectly flat magnetic structure the equilibrium spin vector $\mathbf{n}_{\mathbf{o}}$ is aligned with the vertical magnetic field $\mathbf{B}$ of the dipoles where the radiative Sokolov-Ternov polarizing mechanism originates. Polarization builds up to a maximum value of $8 / 5 \sqrt{3} \sim 92.4 \%$

Misalignments in a realmachine cause the beam to experience non-vertical magnetic fields which bend the spin away from the unperturbed direction and generate spurious vertical dispersion. The equilibrium spin vector becomes orbit-dependent and spin diffusion occurs due to random fluctuations of the precession axis caused by quantized energy changes from emission of synchrotron radiation, resulting in a reduced asymptotic polarization level. Spin diffusion is governed by the spin-orbit coupling function $\boldsymbol{\Gamma}(s)=\gamma \frac{\partial \mathbf{n}(s)}{\partial \gamma}[7]$ and the reduced asymptotic polarization level can be written as :

$$
P_{\perp}^{\infty}=\frac{8 / 5 \sqrt{3}}{1+\left(\frac{\tau_{p}}{\tau_{d}}\right)_{\text {orbit }}}
$$

where both the polarization and the depolarization rates

$$
\left(\tau_{p}\right)^{-1} \propto \oint \frac{d s}{|\rho|^{3}} \quad, \quad\left(\tau_{d}\right)^{-1} \propto \oint \frac{|\boldsymbol{\Gamma}|^{2}}{|\rho|^{3}} d s
$$

are driven by the synchrotron radiation term $\oint \frac{d s}{|\rho|^{3}}$. 


\subsubsection{Harmonic Spin Matching}

Spin motion can be highly perturbed when the spin precession is in phase with energy (synchrotron) and betatron oscillations, in particular for harmonics of the perturbed orbit close to the actual spin tune. Suggested [4] in 1985, Harmonic Spin Matching (HSM) is a method to minimize the spurious tilt of the spin precession axis by compensating the harmonics of the Fourier expansion of the real orbit close to a specific value of the spin tune. The method relies on a lengthy procedure for the correction of the orbits, made difficult from the tiny entities of the corrections required, only monitored by the observation of the effects on the polarization itself.

The HSM implementation described in [5] was intended to reduce the drawback caused by the very long polarization time in LEP and consisted in deriving the amplitude of the correcting bumps for the harmonics of interest directly from the beam position information (Deterministic $H S M$ ).

Its application proved to be very beneficial allowing to improve the polarization level obtained with the solenoid compensation $(\sim 15 \%)$ to more than $40 \%[1]$.

A further improvement of the $H S M$ method was tried by varying the correcting harmonic amplitudes in steps while recording the polarization level to search for an optimum (Empirical $H S M$ ). The experiment, performed at a spin tune $\nu_{s}=101.5$ corresponding to a PEAK-2 CM energy, produced a record level of $(57 \pm 3) \%$ as shown in fig.1.

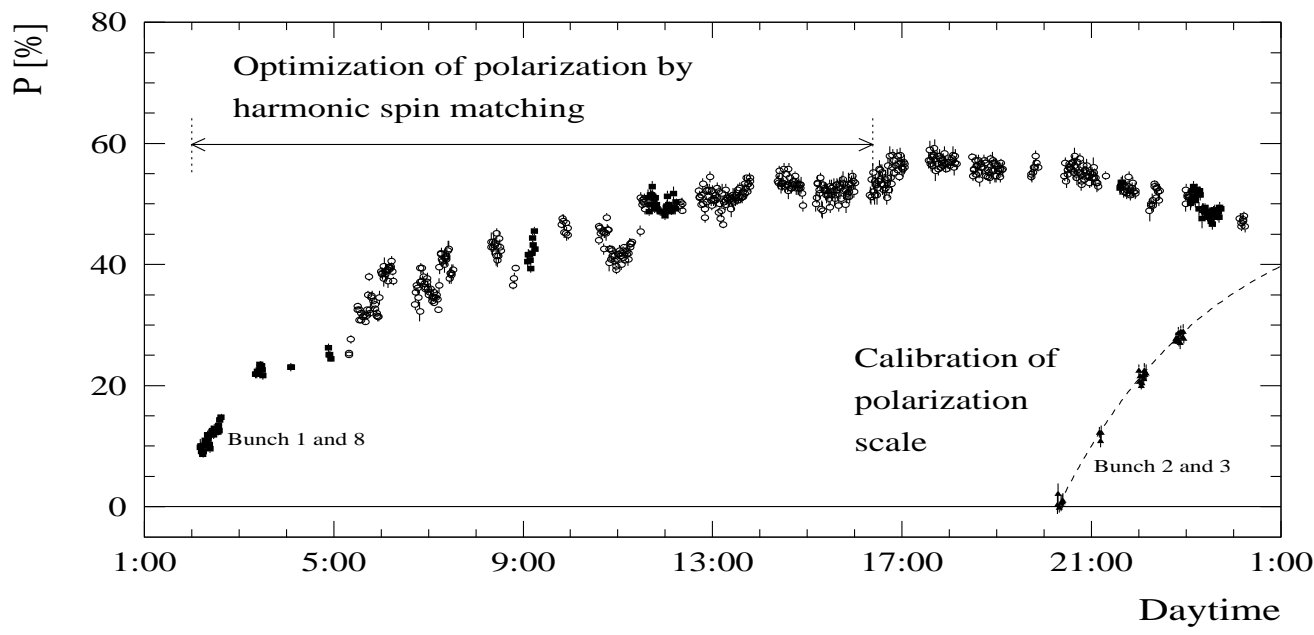

Figure 1: Maximum measured polarization level of $(57 \pm 3) \%$ at PEAK-2 CM energy with the adoption of Deterministic and Empirical Harmonic Spin Matching. Bunches 2 and 3 were on purpose depolarized to calibrate the polarimeter scale by measuring the Sokolov-Ternov radiative polarization rise time.

\section{PRECISE BEAM ENERGY CALIBRATION}

Operational beam Energy Calibration by resonant depolarization [8] was successfully implemented to improve the measurements of the $Z$-resonance mass and width [9] during the energy scan in 1993. A typical example of a spin-tune scan associated to a controlled resonant depolarization is shown in fig.2. 
The limitations and the systematic errors which can affect the Energy Calibration have been thoroughly accounted for in [9] and summarized in table 1. To combine the 1993 Energy Calibration results the measurements were corrected to a reference set of parameters where the effects from terrestrial tides [10] and from temperatures changes in the LEP dipoles were accounted for.

Analysis of the orbits measured during all LEP physics fills [11] allowed the energies measured over 24 calibrations in the 1993 Physics Run to be also corrected for non-periodical ring deformations from, for example, hydrostatic pressure on the tunnel walls due to rain and changes in the water level of the Geneva lake [12].

The time evolution of the electron beam energy during the 1993 scan is shown in fig. 3 .

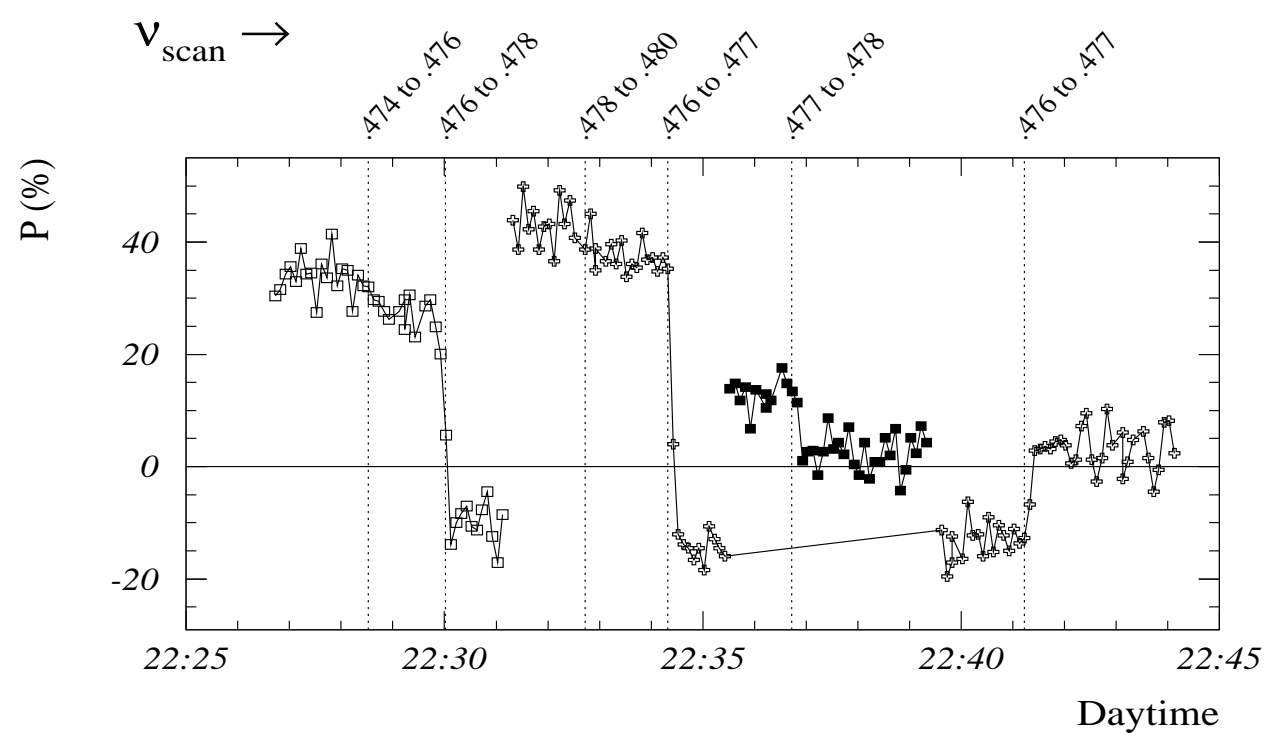

Figure 2: Example of Energy Calibration with Resonant Depolarization. The upper scale shows the non-integer part of the spin tune corresponding to the sharp drop in the polarization level at the depolarizing resonance. A partial spin-flip was checked to be real by flipping it back at the same spin tune $(0.476-0.477)$.

\subsection{Comparing Electron and Positron Beam Energies}

Possible differences between electron and positron beam energies $E_{b}$ were investigated by almost simultaneously calibrating the two beams with a modified setup of the polarimeter to illuminate both beams with the same laser beam back-reflected by a retro-focusing concave mirror [5]. Results shown in fig. 4 are consistent with an energy difference in agreement with the upper limit of $\Delta E_{b} \leq 0.2 \mathrm{MeV}$ from theoretical calculations [13].

The absolute calibration of the beam energies of the off-peak points was determined with a precision of $2 \cdot 10^{-5}$ resulting in a systematic error of $\sim 1.4 \mathrm{MeV}$ on the $Z$-mass and of $\sim 1.5 \mathrm{MeV}$ on the $Z$-width [11]. 


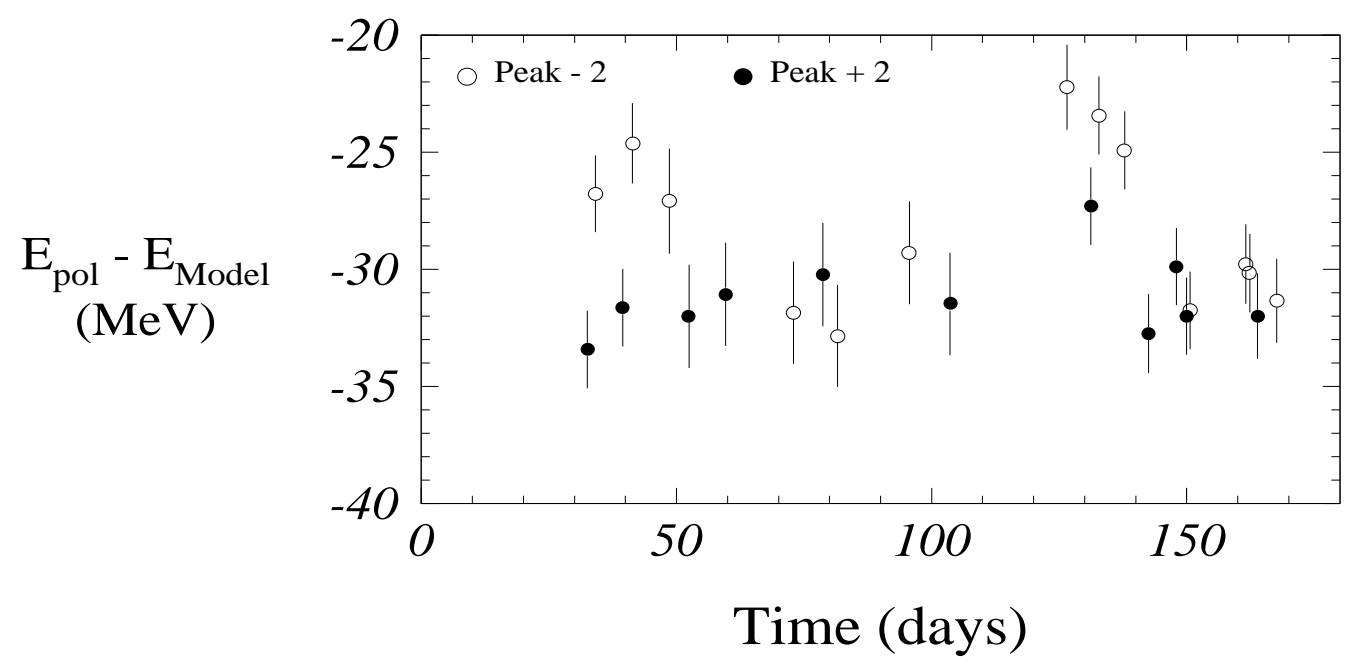

Figure 3: Evolution of the electron beam energy during the 1993 scan after correction of periodic effects (tides), dipole temperature changes and radial orbit movements.
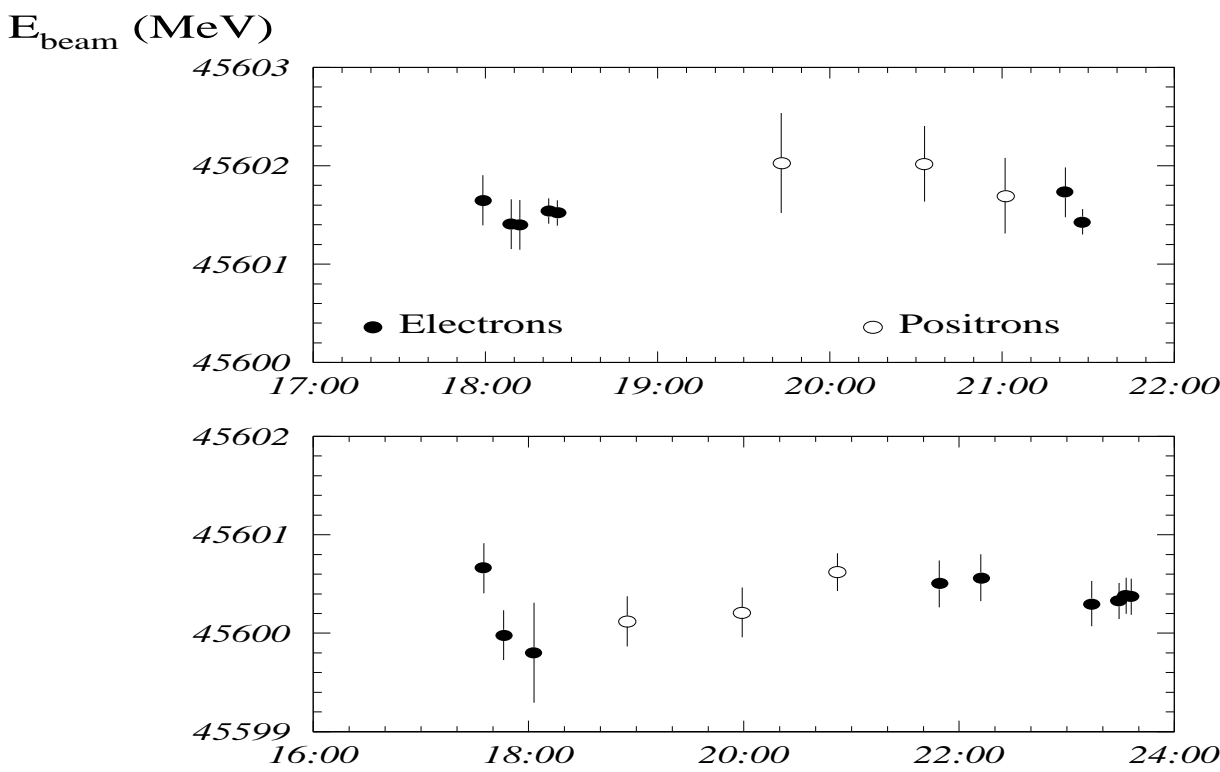

Daytime

Figure 4: Electron and Positron beam energies measured almost simultaneously in two experiments performed on July $15^{\text {th }}$ (top) and August $1^{\text {st }} 1994$. The energies are corrected for tides, magnet temperatures, reference magnet field and slow ring distortions. The resulting energy difference is $\Delta E_{b} \leq 0.2 \mathrm{MeV}$. 


\begin{tabular}{||l|c|c||}
\hline \multicolumn{1}{|c|}{ SOURCE } & \multicolumn{1}{c|}{$\Delta E$} & $\Delta E$ \\
& Theoretical estimates & Experimental upper bound \\
\hline \hline Electron mass & & $13 \mathrm{keV}$ \\
\hline Revolution frequency & & $<1 \mathrm{keV}$ \\
\hline Depolarizer frequency & & $100 \mathrm{keV}$ \\
\hline \hline Width of excited resonance & & $100 \mathrm{keV}$ \\
\hline Interference of resonances & & $<100 \mathrm{keV}$ \\
\hline Quadratic nonlinearities & $<5 \mathrm{keV}$ & $<500 \mathrm{keV}$ \\
\hline$\nu_{s}$-shifts from long. fields & $<5 \mathrm{keV}$ & $<500 \mathrm{keV}$ \\
\hline$\nu_{s}$-shifts from rad. fields & $<100 \mathrm{keV}$ & $<800 \mathrm{keV}$ \\
\hline \hline Total systematic error & $\mathbf{1 0 0} \mathbf{~ k e V}$ & \\
\hline \hline Total upper bound & & $<\mathbf{1 . 1} \mathbf{~ M e V}$ \\
\hline
\end{tabular}

Table 1: Systematic errors in the measurement of the beam energy by resonant depolarization assuming a well corrected vertical orbit. Quoted errors, evaluated at $E=45.6 \mathrm{GeV}$, are understood to be Gaussian and refer to the energy of a single beam. The contributions in the third column are experimental upper bounds used to compute the total upper bound on the systematic error.

\section{POLARIZATION IN COLLISION}

Two experiments were performed to assess the feasibility of achieving transverse polarization with colliding beams. A high transverse polarization level (40\%) was maintained while colliding one $\mathrm{e}^{+}$against one $\mathrm{e}^{-}$bunch in the OPAL detector with high luminosity (beambeam tune shift of $\sim 0.04$ ) at a PEAK-2 CM energy.

Writing the asymptotic value for the observed polarization level as

$$
P_{\perp}^{\infty}=\frac{8 / 5 \sqrt{3}}{1+\left(\frac{\tau_{p}}{\tau_{d}}\right)_{\text {or } b i t}+\left(\frac{\tau_{p}}{\tau_{d}}\right)_{B B}}
$$

one can phenomenologically distinguish between the depolarizing effects inherent to the quality of the orbit in the arcs and those produced by the beam-beam interaction.

The contribution from vertical orbit deviations $y_{r m s}$ in the lattice quadrupoles scales as $\left(\tau_{p} / \tau_{d}\right)_{\text {orbit }} \propto|\boldsymbol{\Gamma}|^{2} \propto y_{r m s}^{2}$ (4) and the chance to produce high transverse polarization levels relies on the quality of the vertical orbit in the arcs.

The contribution from beam-beam interaction can be reduced with a proper choice of machine tunes (betatron and synchrotron) once the beam energy has been correctly defined to avoid single beam depolarizing resonances.

The experiments were performed at a beam energy corresponding to a PEAK-2 CM energy $\left(\nu_{s} \cong 101.5\right)$ in order to avoid systematic spin resonances of the type (1).

A $3 e^{-}$ON $1 e^{+}$bunch scheme [14] allowed colliding at one IP at a time and to measure the polarization level for colliding and non-colliding bunches as shown in table 2. Only the bunches $P 1, E 1, E 2, E 3$ were injected to produce collisions between $P 1$ and $E 2$ in $I P 6$ (and later in $I P 2$ ). The non-colliding $\mathrm{e}^{-}$bunches $E 1$ and $E 3$ were used for Energy Calibration and to provide a reference value for "single beam" polarization.

The results are reported in the following. 


\begin{tabular}{||c|c|c||}
\hline IP & ENCOUNTERS & MODE \\
\hline $1 \& 5$ & P1·E1 + P2·E2 + P3·E3 + P4·E4 & V-separation \\
\hline $2 \& 6$ & P1·E2 + P2·E3 + P3·E4 + P4·E1 & collisions \\
\hline $3 \& 7$ & P1·E3 + P2·E4 + P3·E1 + P4·E2 & V-separation \\
\hline $4 \& 8$ & $\mathrm{P} 1 \cdot E 4+$ P2·E1 + P3·E2 + P4·E3 & (collisions $)$ \\
\hline
\end{tabular}

Table 2: Collisions with 4 equidistant bunches per beam. In thick, the bunches colliding with the adopted scheme.

\subsection{The first Experiment}

$4 \times 220 \mu \mathrm{A}$ were injected and accelerated at the betatron/synchrotron tunes (2) and the beam energy measured to localize the working point in the spin-tune space to avoid depolarizing resonances.

The quality of the vertical orbit was not very satisfactory $\left(y_{\mathrm{rms}}=0.45 \mathrm{~mm}\right)$ and the application of Deterministic $H S M$ did not help in reaching a polarization level higher than $\sim 25 \%$ for the non-colliding bunch $E 3$.

The polarization level for the $E 2$ bunch, when colliding in $I P 6$ with the $P 1$ bunch, remained limited to $\sim 20 \%$, but didn't decrease when the two bunches were made to collide also in IP2 (see fig.5).

The luminosity measured in one of the LEP detectors (OPAL) was consistent with a relatively high beam-beam strength parameter:

$$
L_{O P A L}=(1.3 \rightarrow 1.0) \times 10^{30} \mathrm{~cm}^{-2} \mathrm{~s}^{-1} \quad, \quad \xi_{\mathrm{y}} \sim 0.037 .
$$

\subsection{The Second Experiment}

$4 \times 190 \mu \mathrm{A}$ were accelerated in LEP with the same machine conditions as in the previous experiment, with the exception that this time the four solenoids were switched OFF. A reasonable amount of time was spent trying to improve the polarization level with separated beams adopting a kind of educated HSM [15] in which the true bump amplitude needed to compensate the four harmonics was experimentally determined by observing the separated effect of each of them. As a result the polarization level started rising from a saturation value of $\sim 25 \%$ to more than $30 \%$ (see fig. 6 ) and kept rising when $E 2$ was brought in collision with $P 1$ in OPAL. The empirical HSM procedure was pursued with colliding beams and a $40 \%$ polarization level was attained for both the colliding and the non-colliding $e^{-}$bunches. High luminosity and beam-beam strength parameter were again measured in OPAL:

$$
L_{O P A L}=(1.5 \rightarrow 1.0) \times 10^{30} \mathrm{~cm}^{-2} \mathrm{~s}^{-1} \quad, \quad \xi_{\mathrm{y}} \sim 0.040 .
$$

When a second interaction was added in IP2 the polarization level showed a tendency to decrease, but the beams were lost due to a machine problem before we succeeded in compensating the effect.

\subsection{Summary of Results}

The results obtained in the two experiments described above are compared to the high polarization one obtained at the same beam energy in 1993 [5] in table 3. 


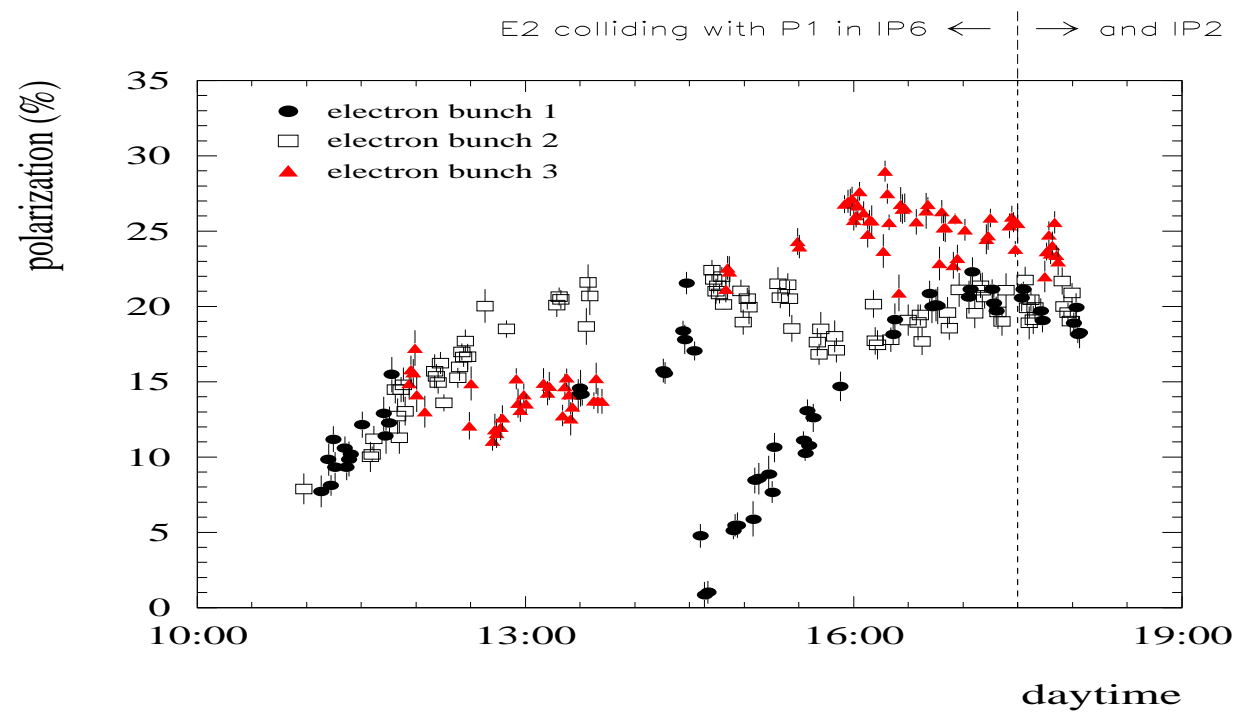

Figure 5: Polarization with colliding beams. With the adoption of a purely Deterministic $H S M$ scheme the maximum polarization level attained by the non-colliding electron bunch $E 3$ was about $25 \%$ while the colliding bunch $E 2$ reached a maximum of $\sim 20 \%$. The electron bunch $E 1$ was depolarized on purpose to calibrate the polarimeter scale through the SokolovTernov polarization rise time.

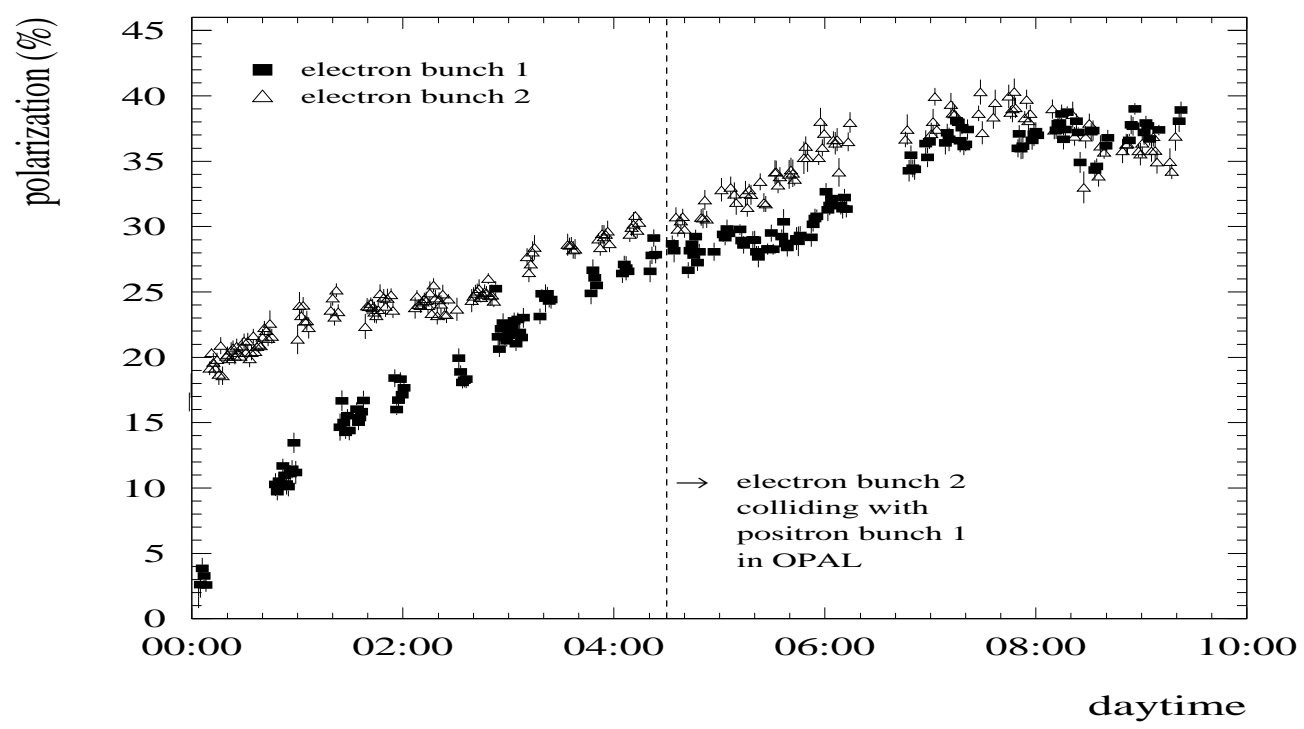

Figure 6: Polarization for colliding and non-colliding electron bunches was raised up to a $40 \%$ level during the last experiment in the 1994 LEP Run with the adoption of Empirical and Deterministic $H S M$ which allowed for polarization to keep rising after the E2 electron bunch was made to collide with the positron bunch $P 1$ in the OPAL experiment. 
In particular it is shown (fifth column) that the beam-beam depolarization rate measured during the second experiment was at least a factor of three smaller than the polarization one.

\begin{tabular}{|c|c|c|c|c|c|c|}
\hline Date & MODE & $P_{\infty}$ & $\left(\tau_{p} / \tau_{d}\right)_{\text {orbit }}$ & $\left(\tau_{p} / \tau_{d}\right)_{B B}$ & $y_{r m s} / m m$ & $\xi_{y}$ \\
\hline 23.08 .93 & 1 beam & $57 \%$ & 0.62 & - & 0.33 & - \\
\hline 16.11 .94 & $\begin{array}{c}2 \text { beams, Sep. } \\
\text { Colliding }\end{array}$ & $\begin{array}{l}26 \% \\
22 \% \\
\end{array}$ & 2.55 & 0.65 & 0.45 & 0.037 \\
\hline 05.12 .94 & $\begin{array}{l}2 \text { beams, Sep. } \\
\text { Colliding }\end{array}$ & $\begin{array}{l}40 \% \\
38 \%\end{array}$ & 2.7 & $\leq 0.3$ & 0.36 & 0.040 \\
\hline
\end{tabular}

Table 3: Comparison between the experiments 1994 and the High Polarization experiment in 1993. All experiments performed at PEAK-2 CM energy and with HSM.

\section{FUTURE PLANS}

\subsection{Energy Calibration in Collision}

Differently from the Energy Calibration campaign for the 1993 Scan [11] the proposed 1995 schedule foresees to devote between 20 to $40 \mathrm{pb}^{-1}$ to perform Energy Calibration at both PEAK \pm 2 energies [16],[17]. With en expected 1995 performance of about $1 \mathrm{pb}^{-1}$ per fill, about 40 fills in the 11 weeks between July and September should be calibrated.

A strong motivation exists then to perform the calibration during the LEP operation for physics. To meet this target some problems will be addressed [14] namely :

1. the polarization $\beta$-tron tunes (2) differ from those used in "production";

2. the present $H S M$ scheme affects the Luminosity and proved to be marginal with the optics used at the end of the 1994 run.

\subsubsection{Residual Longitudinal Polarization at IP's}

The possibility that a small amount of residual longitudinal polarization at the Interaction Points could survive due to machine optics imperfections is of some concern for the LEP experimental collaborations since it represents a bias in the measurements.

The question was addressed in [18] where, in the hypothesis that the experimental solenoids are spin-compensated, the main source for this phenomenon is traced back to beam orbit offsets in the low- $\beta$ doublets close to the IP's, which can behave like small spin rotators. Quantitatively, a $1 \mathrm{~mm}$ orbit deviation in the strong focusing doublets can produce $\sim 40$ mrad local rotation of the $\mathbf{n}_{0}$ vector which corresponds to a $\sim 0.4 \%$ longitudinal polarization level at the experimental IP's for a $10 \%$ transverse polarization.

This value, well within the limits acceptable by the experiments, can be drastically reduced centering the beams in the low- $\beta$ doublets by means of the $K$-modulation technique [19]. Under these conditions, the effect of residual longitudinal polarization at the Interaction Points leads to negligible systematic uncertainties on precision measurements [20].

\section{CONCLUSIONS}

Considerable achievements have been obtained with transverse polarization at LEP in the last two years, both with single-beam operation and with colliding beams, thanks to the 
successful implementation of techniques intended to compensate depolarizing effects from the experimental solenoids and to reduce the contribution to spin diffusion from machine imperfections and large closed orbit deviations.

The Energy Calibration campaign scheduled for the 1995 LEP Run is expected to absorb a considerable amount of beam time during the operation for physics.

The encouraging results obtained with polarization in collision at a PEAK-2 beam energy in 1994 are being considered to be adapted to a more efficient application of the calibration procedures in the 1995 scan to reduce the $Z$-production time to invest into Energy Calibration.

\section{References}

[1] Assmann, R. et al., Polarization Studies at LEP in 1993, CERN SL/94-08, March 1994.

[2] Blondel, A., Compensation of Integer Spin Resonances Created by Experimental Solenoids, LEP Note 629 (1990).

[3] Grote, H., A New Solenoid Compensation for Polarization compatible with Pretzel, SL/Note 94-128 (AP), July 1994.

[4] Rossmanith, R. and Schmidt, R., Compensation of Depolarizing Effects in Electron Positron Storage Rings, NIM A 236 (1985) 231.

[5] Assmann, R., Transversale Spin-Polarisation und ihre Anwendung für Präzisionsmessungen bei LEP, PhD Thesis, 1994.

[6] Koutchouk, J.-P., 8th. H. E. Spin Physics Symposium, AIP Conf. Proceedings No.187, Minneapolis, MN, 1988.

[7] Ya. S. Derbenev and A. M. Kondratenko, Sov. Phys. JETP 37 (1973), 968.

[8] Arnaudon, L., et al., Measurement of LEP beam energy by Resonant Spin Depolarization, Phys. Lett. B 284 (1992) 431-439.

[9] The LEP Polarization Team, Accurate Determination of the LEP Beam Energy by Resonant Depolarization, CERN SL/94-71 (BI) August 1994.

[10] The LEP Polarization Team, Effects of Terrestrial Tides in the LEP Beam Energy, (Submitted to NIM A).

[11] The LEP ENERGY Working Group, The Energy Calibration of LEP in the 1993 Scan, CERN SL/95-02 and CERN PPE/95-10, Feb. 1995.

(Submitted to Zeitschrift für Physik C).

[12] Wenninger,J., Radial Deformations of the LEP ring, CERN SL/Note 95-21 (OP), Feb. 1995.

[13] Drees, A., Energy Difference of Electron and Positron Beam, CERN SL/Note 94-100 (BI), March 1995.

[14] Placidi, M., Transverse Polarization in Collision at LEP, Vth Chamonix Workshop on LEP Performance, Chamonix, 13-18 January 1995.

[15] Blondel, A., Report in publication. 
[16] Wenninger, J,. What will cost in time and efficiency in order to calibrate the LEP energy every fill?, Vth Chamonix Workshop on LEP Performance, Chamonix, 13-18 January 1995.

[17] Camporesi, T., Overall requirements for LEP in 1995 (and 1996), Vth Chamonix Workshop on LEP Performance, Chamonix, 13-18 January 1995.

[18] Assmann, R. and Koutchouk, J.-P., Residual Longitudinal Polarization at LEP, CERN SL Note 94-37, April 1994.

[19] Dehning, B., Do we need K-modulation in the arcs?, Vth Chamonix Workshop on LEP Performance, Chamonix, 13-18 January 1995.

[20] Blondel, A., Systematic Uncertainties on LEP Electroweak observables due to Unknown Residual Longitudinal Beam Polarization, ALEPH Note 95-018, Feb. 1995. 